\title{
Primary Renal Primitive Neuroectodermal Tumor/Ewing's Sarcoma Imaging and Pathologic Findings of a Patient with a Nine Year, Eight Month Disease Free Period: Case Report and Review of Literature
}

\author{
Ivan Zokalj,,$^{, *}$ Jasminka Igrec, ${ }^{1}$ and Antonio Plesnar ${ }^{2}$ \\ ${ }^{1}$ Department of Radiology, County Hospital Cakovec, Cakovec, Croatia \\ ${ }^{2}$ Department of Surgery, Division of Urology, County Hospital Cakovec, Cakovec, Croatia \\ "Corresponding author: Ivan Zokalj, Department of Radiology, County Hospital Cakovec, Cakovec, Croatia. E-mail: izokalj@gmail.com
}

Received 2016 March 18; Accepted 2016 April 30.

\begin{abstract}
Introduction: Primitive neuroectodermal tumor (PNET)/Ewing's sarcoma (EWS) belongs to a family of neoplasms that are presumed to originate from the neuroectodermal crest. PNET/EWSs are highly aggressive malignancies that usually present in the form of bone or soft tissue masses and usually affect adolescents and young adults. Primary PNET/EWS of the kidney is very rare.

Case Presentation: We present the case of a 32-year-old female patient with primary renal PNET/EWS diagnosed nine years and eight months earlier. The patient presented with acute flank pain in the left lumbar region, hematuria, and episodes of high body temperature of $40^{\circ} \mathrm{C}$. Abdominal ultrasound (US) and subsequently performed computed tomography (CT) revealed a large renal mass of heterogenous structure. The kidney tumor had central necrotic hypodense areas and strongly peripherally enhanced solid parts on postcontrast CT images. Immunohistochemistry revealed positivity for CD99 and neuron-specific enolase (NSE). Tumor cells were negative for $\mathrm{CD} 3, \mathrm{CD} 20$, chromogranin, synaptophysin, vimentin, and neurofilament. Reverse transcription polymerase change reaction (RT-PCR) revealed EWS/FL1 translocation type 2. The patient underwent nephrectomy and polychemotherapy. The follow-up nine years and eight months after the diagnosis showed no evidence of tumor.

Conclusions: PNET/EWS should be included in the differential diagnosis of renal tumors in symptomatic young adults. Patients with localised PNET/EWS treated with a combination of surgery and chemotherapy have an excellent chance of long-term survival, as in the case we have presented.
\end{abstract}

Keywords: Tumor, Kidney, Primitive Neuroectodermal Tumor, Ewing's Sarcoma

\section{Introduction}

Primitive neuroectodermal tumor (PNET) is a member of the Ewing's sarcoma/primitive neuroectodermal tumor family of tumors (1). Based on the assumed etiology of these tumors, PNET arises from the neural crest $(2,3)$. ET/EWS tumors have the same chromosomal translocation $[\mathrm{t}(11 ; 22)$ ( $\mathrm{q} 24 ; \mathrm{q} 12)]$ registered in $90 \%$ of cases (2). PNETs are malignant tumors composed of small, round cell neoplasms that usually present in the form of a bone or soft tissue mass. Peripheral PNETs are usually located in the chest wall and paraspinal regions and less frequently in the genitourinary system (3-5). Renal PNET (rPNET) is a very rare form of ES/PNET tumor that predominantly occurs in older children and adolescents $(4,5)$. Renal PNETs are usually presented in the literature in the form of case studies and meta-analyses. The five-year disease free survival rate is about $45 \%$ - 55\% (6). We report the case of a 32-yearold female patient with renal PNET diagnosed nine years and eight months previously, treated with nephrectomy and chemotherapy, and followed up with imaging meth- ods that revealed no signs of disease.

\section{Case Presentation}

We present the case of 32-year-old female patient who presented to the urologist with fever lasting for the previous two months, usually $37^{\circ} \mathrm{C}$ to $38^{\circ} \mathrm{C}$, with occasional temperature leaps to $40^{\circ}$. Laboratory blood tests showed leukocytosis with cell counts up to 19,000. The patient did not complain of lumbar pain. Urinalysis showed leukocyturia, erythrocyturia, and slight positivity for protein. The patient was treated with a combination of amoxicillin, clavulonic acid, and cefuroxime. Abdominal ultrasound and multiphase CT depicted a large, expansive, heterogenous mass in the lower pole of the left kidney measuring $92 \times 59 \times 76 \mathrm{~mm}$. CT scans showed a renal neoplastic mass with increased peripheral inhibition and poor central inhibition, possibly due to central necrosis. The tumor was unsharply demarcated from the surrounding parenchyma and pelvic system (Figure 1). Regional lymphadenopathy was present, but there were no signs of 
propagation in the main renal vein or perinephric fat tissue, and there were no signs of liver or bone metastasis. Chest CT showed no signs of lungs metastasis or mediastinal lymphadenopathy. In order to determine the final histopathological diagnosis, histopathological and immunohistochemical analyses were performed, complemented by reverse transcryptase-polymerase change reaction (RT-PCR). Gross examination of the slitted specimen showed a sharply circumscribed tumor measuring $9 \times 6$ $\times 6 \mathrm{~cm}$ and replacing a large part of the kidney. The renal tumor displayed infiltrated parenchyma but no expansion into the pelvic system and ureter or signs of spreading into the main renal vein. Gross appearance of the tumor was white-yellow with large necrotic areas. The microscopic analysis of perinephric fat tissue, regional lymph nodes, and the left adrenal gland revealed no signs of metastasis. Microscopic analysis showed that the tumor was composed of solid nests and stripes of tumor cells with small oval and round nuclei, and some of the nuclei contained small nucleoli (Figure 2). The tumor cell cytoplasm was scanty and pink with coarser appearance of chromatin. Mitotic figures were also found. Immunohistochemical analysis included MIC2 gene product/CD 99 (Figure 3), neuron-specific enolase (NSE) (Figure 4), CD3, CD 20 , chromogranin, synaptophysin, vimentin, and neurofilament. Immunohistochemical staining revealed positive expression of CD99 and NSE in all tumor cells. The tumor cells did not stain with CD3, CD 20, chromogranin, synaptophysin, vimentin, or neurofilament. RT-PCR analysis for EWS-FLI1 fusion proteins was positive for type II $191 \mathrm{pb}$ and negative for type I $125 \mathrm{pb}$. Diagnosis of rPNET set on the basis of characteristic morphological characteristics and results of immunohistochemical analysis was confirmed by RT-PCR. After nephrectomy, the patient received 14 cycles of a VAC/IE regiment adjuvant chemotherapy comprised of vincristine, doxorubicin, cyclophosphamide, ifosfamide, and etoposide. Our patient was disease-free for nine years and eight months. Blood and urine laboratory test values were in the normal range.

The study was approved by the institutional ethical committee and conducted according to the principles of the declaration of Helsinki of the world medical association. The authors declare that they have no conflicts of interest. The authors did not receive any grants for writing this article.

\section{Discussion}

Primary renal PNET is a very rare member of the PNET/EWS family of tumors, which to the best of the authors' knowledge was first described in 1975 by Seemayer
Figure 1. An axial CT Image in the Nephrographic Phase Depicts a Large, Expansive Mass in the Heterogenuous Structure of the Left Kidney and Enlarged Retroperitoneal Lymph Nodes

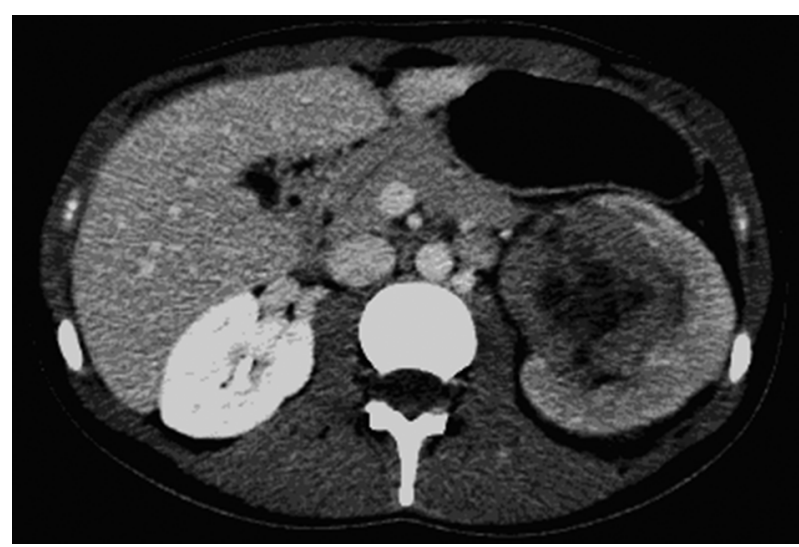

The renal expansive process had higher attenuation values peripherally, and lower values centrally, most likely indicating necrotic parts.

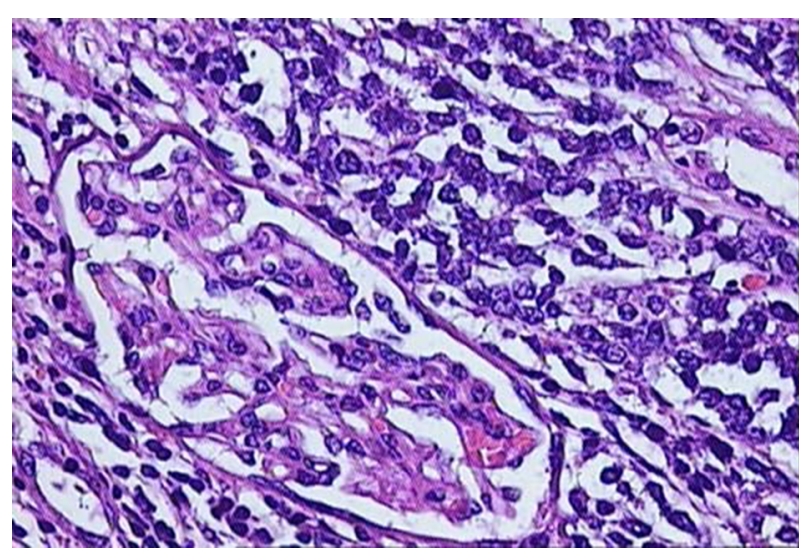

Figure 2. Hematoxylin and Eosin Staining (Magnification, X400) of rPNET Shows Renal Parenchyma Infiltrated With Tumor Tissue Consisting of Solid Nests and Stripes of Tumor Cells With Small Oval and Round Nuclei and Scant Cytoplasm (Courtesy of Mirjana Čačić, M.D., Ph.D.)

et al. (7). The possible etiology of rPNET includes the theory of neural cell intussusception in the kidney during development, the theory of adrenergic fibers that invert into the kidney from the celiac plexus, and the theory of neural crest cells that have migrated to the kidney and begun tumorigenesis (1). Renal PNET most commonly affects children and young adults, with a mean age of 30.4 years and a slight male predominance of $61 \%(1,3,6)$. Primary rPNETs are usually very aggressive tumors with distant metastasis and local reccurence in more than $50 \%$ of patients (5). The overall five-year disease-free survival rate for patients with peripheral PNETs is about 45\% - 55\% in patients with tumors localized in the kidney (5). Patients with rPNET di- 


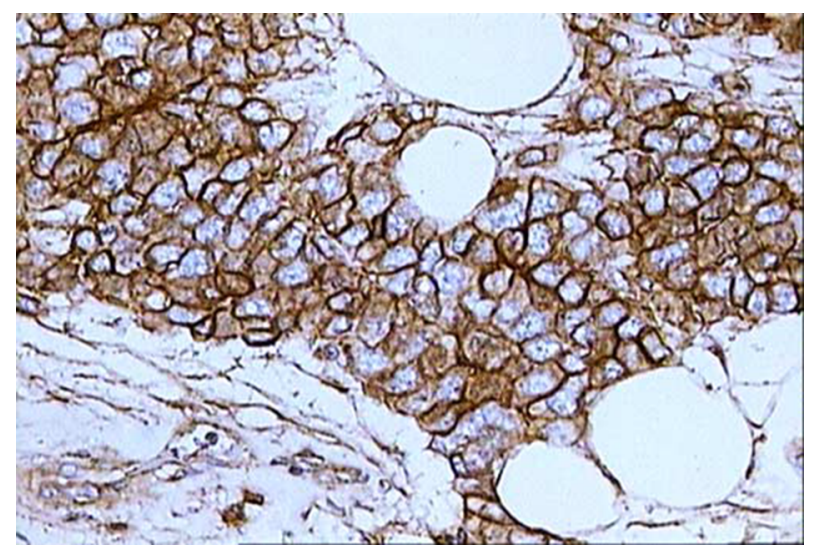

Figure 3. Immunohistochemical Staining of the MIC2 Gene Product (Magnification, $\mathrm{X} 400$ ) of rPNET Shows Strong Mebrane Positivity of the Tumor Cells (Courtesy of Mirjana Čačić, MD, PhD)

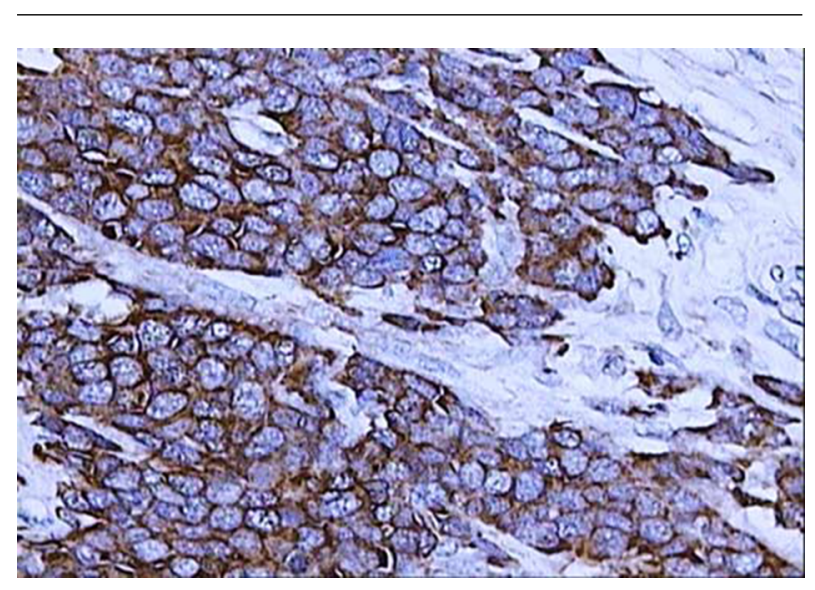

Figure 4. Immunohistochemical Staining of rPNET (Magnification, X400) Revealed the Strong Positive Expression of NSE in All Tumor Cells (Courtesy of Mirjana Čačić, $\mathrm{MD}, \mathrm{PhD}$ )

agnosed in the advanced stage have a median relapse-free survival of two years (5). Renal PNET can present in various forms with symptoms such as malaise, nephric colic, flank pain, testicular pain, dysuria, dyspnea, dizziness, and clinical signs such as an increase in abdominal circumference, weight loss, a palpable mass, night sweats, fever, hematuria, and varicocele (6). Primary renal PNETs are usually large tumors with a diameter of $>10 \mathrm{~cm}(8,9)$. Our patient had a less common pattern of presentation, she was subfebrile and she had hematuria and no pain in the left lumbar region at the time of diagnosis.

The radiographic features of renal PNETs presented in previously published articles mainly case reports include large renal masses of heterogeneous structure with areas of internal hemorrhage, necrosis, and diffuse large cal- cification but no signs of extensive parenchyma infiltration (6, 9-11). The same radiological pattern had a tumor formation in our patient's case. The histological characteristics of renal PNET at the light microscopic level are small, uniform round or oval cells with dark nuclei, and in some cases vesicular nuclei and nucleoli can be observed. In most of the cases of rPNET, numerous mytotic figures can be detected. The renal PNET tumor cells commonly contain a minimal amount of cytoplasm within illdefined borders $(1,3,12)$. The tumor cells of renal PNETs can form clusters with a neurofibrillary stromal core and pseudorosettes (Homer-Wright rossetes), which are considered the most diagnostically useful histological feature. However, pseudorosettes are also visible in neuroblastomas (12). Histological differential diagnoses for renal PNET include the „round cell“ tumors of the kidney, such as blastema-predominant Wilm's tumor, lymphoma, clear cell sarcoma, small cell carcinoma, monophasic poorly differentiated synovial sarcoma, neuroblastoma, desmoplastic round cell tumor, rhabdoid tumor, and rhabdomyosarcoma (1, 3-5, 12). Differential diagnostic problems can be partially resolved with immunohistochemistry $(1,3)$. MIC2 gene product or CD99 antigen (Cluster of differentiation 99 antigen) is a monoclonal antibody that recognizes the glucoprotein p 30/32. Diffuse membrane positivity on CD99 can be detected in almost all reported cases of PNET, although it is not an absolute biomarker. The specificity of CD99 for diagnosis of PNET has been questioned, especially for differentiation from small cell carcinoma, Wilm's tumor, and non-Hodgkin's lymphoma (1, $3,4,13,14)$. Neuron-specific enolase (NSE) is positive in 95\% of cases of rPNETs, although it can be positive in neuroblastoma as well $(1,3)$. Other rPNET commonly positive immunohistochemical markers are S-100, synaptophysin, Leu-7, and vimentin, positive in $48 \%$ to $70 \%$ of cases (5). The samples in the present case are positively stained on CD99 and NSE, as in most cases of rPNET, but negative for vimentin and synaptophysin, which is atypical for rPNET. WT-1 is an associated tumor supressor gene that is overexpressed in Wilm's tumor but not in PNET/EWS. Positive results of immunochemistry for the carboxy-terminus of Friend leukemia integration 1 proto-oncogene (FLI-1) proteins on tumor cells is sensitive and highly specific for PNET/EWS. The previously mentioned cytogenetic difference between PNET/EWS and Wilm's tumor cells forms the basis for the immunochemical differential diagnosis with stainings on WT-1 and FLI-1 (3). Chromosomal translocation $\mathrm{t}(11 ; 22)$ ( q 24; q 12) can be found in 90\% - 95\% of renal PNET/EWS; subsequent immunohistochemical staining of the carboxy-terminus of FL1 is sensitive and highly specific for the diagnosis of PNET/EWS, but not characteristic $(2,3)$. The previously mentioned chromosome translocation re- 
sults in the formation of a EWS-FLI1 fusion gene, which encodes a chimeric EWS-FLI-1 protein (2). EWS-ET fusion genes are presumed to be the driving force in Ewing's sarcoma pathogenesis (2). The influence of EWS-FLI1 fusion type on prognosis has not been unequivocally confirmed in previous studies (15). The EWS/FLI1 fusion may be detected directly with the use of RT-PCR or fluorescence in situ hybridization (FISH), otherwise indirectly with standard cytogenetic studies by the $\mathrm{t}(11 ; 22)$ (q 24; q 12) (1). The diagnosic sensitivity of the RT-PCR assay in a study performed by Parham et al. was 80\% (1). Our patient had EWS/FLI1 fusion type II detected with RT-PCR, but unlike in most cited studies, the patient presented in this paper had a longer disease-free period.

The combination of surgery and chemotherapy is the most common therapeutic approach for the patient with rPNET $(12,16)$. Complete nephrectomy complemented with resection of other affected organs or vasculature (e.g., cavotomy) is the standard surgical therapy (12). Adjuvant chemotherapy for PNET/EWS includes a VAC/IE chemotherapeutic regimen $(17,18)$. Radiation therapy in rPNET is indicated only in extracapsular spread of tumors and tumor residues $(5,16)$.

Renal PNETs are diagnosed at the advanced stage in 58\% to $66 \%$ of cases $(5,19)$. The overall five-year survival rate for rPNETs is between $42 \%$ and $67 \%(5,16)$. In the study of Le Deley et al. (15), which included 565 patients, the probability of disease progression or relapse was $41 \%$ (95\% CI, $37 \%$ to $46 \%$ ) at five years. In the retrospective study of Risi et al. (19), which included 116 published cases of rPNETs from 1975 to 2012, the median overall survival time was 24 months (95\% CI 4.5 -15.1).

Our patient was disease-free for nine years and eight months. During the first three years of follow-up, the patient was monitored by CT, and during the last six years, the patient was monitored with a combination of conventional chest radiographs and MRI of the abdomen and pelvis. To the best of the authors' knowledge, the longest individual disease-free survival period in patients with published medical records of follow-up was 8.7 years; however, the authors were unable to find specific details about treatment regimens (15).

\subsection{Conclusion}

Renal PNET/EWS should be placed on the list of differential diagnoses in younger symptomatic patients, in whom a variety of diagnostic tests reveal a large tumor mass, because this has a significant impact on the treatment and prognosis. Immunohistochemical and cytogenetic studies play an important role in the diagnosis of rPNET. A combination of complete nephrectomy and ad- juvant chemotherapy with a VAC/IE regimen can achieve good results in the treatment of localized rPNET.

\section{Acknowledgments}

The authors wish to thank Mirjana Čačić M.D., Ph.D. for the valuable assistance given during the preparation of this paper.

\section{Footnote}

Authors' Contribution: Ivan Zokalj performed the patient data and image gathering, carried out the literature review, wrote the manuscript, prepared the figures, and revised the manuscript with coauthors. Jasminka Igrec assisted in the drafting and revision of the manuscript. Antonio Plesnar assisted in the patient data gathering and revision of the manuscript. All authors read and approved the final manuscript.

\section{References}

1. Parham DM, Roloson GJ, Feely M, Green DM, Bridge JA, Beckwith JB Primary malignant neuroepithelial tumors of the kidney: a clinicopathologic analysis of 146 adult and pediatric cases from the National Wilms' Tumor Study Group Pathology Center. Am J Surg Pathol. 2001;25(2):133-46. [PubMed: 11176062].

2. Lin PP, Brody RI, Hamelin AC, Bradner JE, Healey JH, Ladanyi M. Differential transactivation by alternative EWS-FLI1 fusion proteins correlates with clinical heterogeneity in Ewing's sarcoma. Cancer Res. 1999;59(7):1428-32. [PubMed:10197607].

3. Jimenez RE, Folpe AL, Lapham RL, Ro JY, O'Shea PA, Weiss SW, et al. Primary Ewing's sarcoma/primitive neuroectodermal tumor of the kidney: a clinicopathologic and immunohistochemical analysis of 11 cases. Am J Surg Pathol. 2002;26(3):320-7. [PubMed: 11859203]

4. Gonlusen G, Ergin M, Paydas S, Bolat FA. Primitive neuroectodermal tumor of the kidney: a rare entity. Int Urol Nephrol. 2001;33(3):449-51. [PubMed: 12230269].

5. Ellinger J, Bastian PJ, Hauser S, Biermann K, Muller SC. Primitive neuroectodermal tumor: rare, highly aggressive differential diagnosis in urologic malignancies. Urology. 2006;68(2):257-62. doi: 10.1016/j.urology.2006.02.037. [PubMed: 16904430].

6. Hakky TS, Gonzalvo AA, Lockhart JL, Rodriguez AR. Primary Ewing sarcoma of the kidney: a symptomatic presentation and review of the literature. Ther Adv Urol. 2013;5(3):153-9. doi: 10.1177/1756287212471095. [PubMed: 23730330].

7. Seemayer TA, Thelmo WL, Bolande RP, Wiglesworth FW. Peripheral neuroectodermal tumors. Perspect Pediatr Pathol. 1975;2:151-72. [PubMed: 1129029].

8. Ohgaki K, Horiuchi K, Mizutani S, Sato M, Kondo Y. Primary Ewing's sarcoma/primitive neuroectodermal tumor of the kidney that responded to low-dose chemotherapy with ifosfamide, etoposide, and doxorubicin. Int J Clin Oncol. 2010;15(2):210-4. doi:10.1007/s10147-0100031-3. [PubMed: 20186557].

9. Kuczynski AP, Gugelmin ES, Netto RA. [Primitive neuroectodermal tumor of the kidney in children]. J Pediatr (Rio J). 2001;77(1):49-51. [PubMed: 14647620]. 
10. Maccioni F, Della Rocca C, Salvi PF, Manicone AM, Ascarelli A, Longo F, et al. Malignant peripheral neuroectodermal tumor (MPNET) of the kidney. Abdom Imaging. 2000;25(1):103-6. [PubMed:10652933].

11. Ba L, Tan H, Xiao H, Guan Y, Gao J, Gao X. Radiologic and clinicopathologic findings of peripheral primitive neuroectodermal tumors. Acta Radiol. 2015;56(7):820-8. doi: 10.1177/0284185114539321. [PubMed: 25073463].

12. Bing Z, Zhang P, Tomaszewski JE, Maclennan GT. Primary Ewing sarcoma/primitive neuroectodermal tumor of the kidney. J Urol. 2009;181(3):1341-2. doi:10.1016/j.juro.2008.12.029. [PubMed:19157457].

13. Weidner N, Tjoe J. Immunohistochemical profile of monoclonal antibody 013: antibody that recognizes glycoprotein p30/32MIC2 and is useful in diagnosing Ewing's sarcoma and peripheral neuroepithelioma. Am J Surg Pathol. 1994;18(5):486-94. [PubMed: 7513503].

14. Folpe AL, Hill CE, Parham DM, O'Shea PA, Weiss SW. Immunohistochemical detection of FLI-1 protein expression: a study of 132 round cell tumors with emphasis on CD99-positive mimics of Ewing's sarcoma/primitive neuroectodermal tumor. Am J Surg Pathol. 2000;24(12):1657-62. [PubMed: 11117787].

15. Le Deley MC, Delattre O, Schaefer KL, Burchill SA, Koehler G, Hogendoorn PC, et al. Impact of EWS-ETS fusion type on disease progression in Ewing's sarcoma/peripheral primitive neuroectodermal tumor: prospective results from the cooperative Euro-E.W.I.N.G. 99 trial.J Clin Oncol. 2010;28(12):1982-8. doi: 10.1200/JCO.2009.23.3585. [PubMed: 20308673].

16. Miser JS, Kinsella TJ, Triche TJ, Steis R, Tsokos M, Wesley R, et al. Treatment of peripheral neuroepithelioma in children and young adults. JClin Oncol. 1987;5(11):1752-8. [PubMed: 3316513].

17. Grier HE, Krailo MD, Tarbell NJ, Link MP, Fryer CJ, Pritchard DJ, et al. Addition of ifosfamide and etoposide to standard chemotherapy for Ewing's sarcoma and primitive neuroectodermal tumor of bone. $N$ Engl J Med. 2003;348(8):694-701. doi: 10.1056/NEJMoa020890. [PubMed: 12594313].

18. Ladenstein R, Potschger U, Le Deley MC, Whelan J, Paulussen M, Oberlin O, et al. Primary disseminated multifocal Ewing sarcoma: results of the Euro-EWING 99 trial. J Clin Oncol. 2010;28(20):3284-91. doi: 10.1200/JCO.2009.22.9864. [PubMed: 20547982].

19. Risi E, Iacovelli R, Altavilla A, Alesini D, Palazzo A, Mosillo C, et al. Clinical and pathological features of primary neuroectodermal tumor/Ewing sarcoma of the kidney. Urology. 2013;82(2):382-6. doi: 10.1016/j.urology.2013.04.015. [PubMed: 23800653]. 